\section{Early Career Investigator}

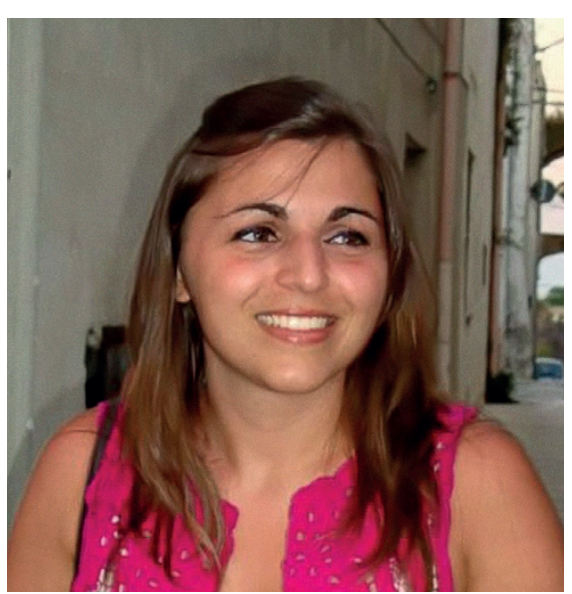

Congratulations to Maria Luisa Tataranno, this issue's Early Career Investigator! A past awardee of the ESPR early investigator exchange program, she describes growing up and attending medical school in southern Italy, her travels to Brazil and Mexico, and her current PhD program in the Netherlands, where she is studying early biomarkers of brain development. In a study reported in this issue, she and her colleagues found that brain activity in preterm infants leads to increased cerebral and cerebellar size. See pages 750 and 834

\section{Head growth in utero predicts sleep patterns in children}

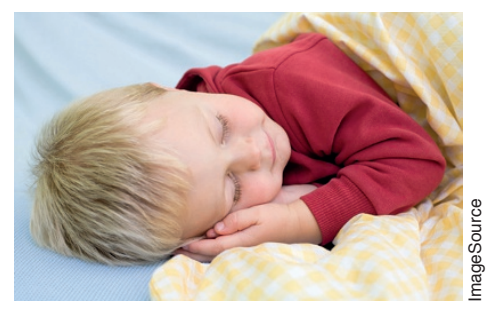

Brain development determines sleeppattern maturation. In this large-scale study, Kocevska et al. obtained fetal ultrasounds for 6,528 children. They found that children with larger head circumference on ultrasound were less likely to be problem sleepers. See page 760

\section{Low maternal SES at birth reduces neurocognition in former ELBWs}

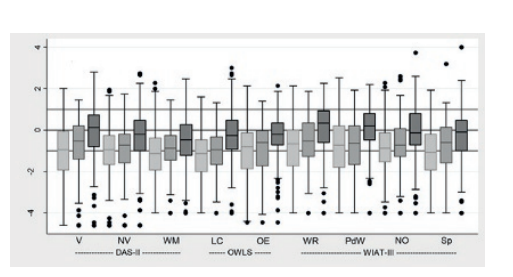

In a study that used maternal educational status as a proxy for socioeconomic status (SES), Joseph et al. showed that extremely-lowbirthweight (ELBW) infants born to mothers with less than 12 years of education had poorer neurocognitive and academic outcomes at 10 years of age. However, maternal educational advancement during a child's first 10 years improved these outcomes. See page 767

\section{Predictors of infant death from sepsis}

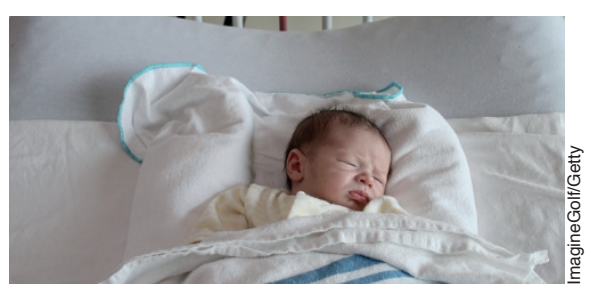

Using data from a clinical trial of zinc in addition to antibiotics for the treatment of sepsis in children, Singh et al. determined that being fed formula or cow's milk, lethargy at presentation, and high levels of c-reactive protein were independent indicators of higher risk of death. See page 784

\section{Outcomes of childhood Goodpasture's disease}

To investigate childhood Goodpasture's disease, which is rare, Menzi et al. obtained questionnaire data from 66 pediatric nephrology centers. Their analysis showed, among

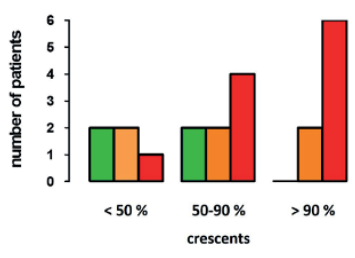

other findings, a lack of pulmonary involvement before puberty and a $50 \%$ rate of progression to end-stage renal disease. See page 813

\section{BCFAs reduce inflammatory markers in enterocytes}

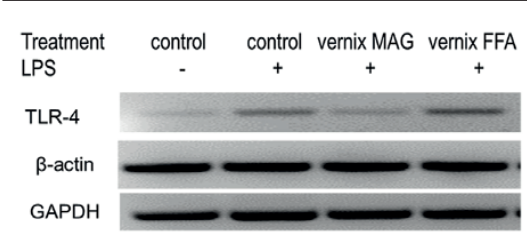

Branched-chain fatty acids (BCFAs), which are high in vernix, have been found to reduce the incidence of necrotizing enterocolitis in an animal model. Yan et al., using an enterocyte cell line, found that BCFAs reduced proinflammatory markers in response to lipopolysaccharide activation. See page 874

\section{PAL may improve outcome of PKU mothers' offspring}

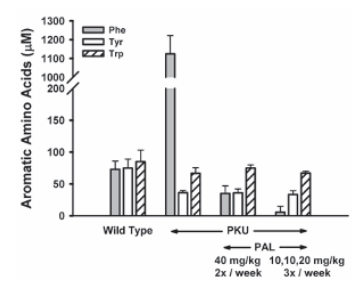

Using an animal model of phenylketonuria (PKU), Zeile et al. treated pregnant mice on a normal diet with phenylalanine ammonia lyase (PAL). Offspring survived to adulthood, in contrast to a high mortality in untreated PKU animals. PAL may be an intervention to improve outcomes of pregnancies in PKU mothers. See page $\mathbf{8 8 9}$ 